\title{
The Scalable Brain Atlas: Instant Web-Based Access to Public Brain Atlases and Related Content
}

\author{
Rembrandt Bakker • Paul Tiesinga • Rolf Kötter
}

Published online: 17 February 2015

(C) The Author(s) 2015. This article is published with open access at Springerlink.com

\begin{abstract}
The Scalable Brain Atlas (SBA) is a collection of web services that provide unified access to a large collection of brain atlas templates for different species. Its main component is an atlas viewer that displays brain atlas data as a stack of slices in which stereotaxic coordinates and brain regions can be selected. These are subsequently used to launch web queries to resources that require coordinates or region names as input. It supports plugins which run inside the viewer and respond when a new slice, coordinate or region is selected. It contains 20 atlas templates in six species, and plugins to compute coordinate transformations, display anatomical connectivity and fiducial points, and retrieve properties, descriptions, definitions and $3 \mathrm{~d}$ reconstructions of brain regions. The ambition of SBA is to provide a unified representation of all publicly available brain atlases directly in the web browser, while remaining a responsive and light weight resource that
\end{abstract}

Rolf Kötter sadly passed away on June 9th, 2010. He co-initiated this project and played a crucial role in the design and quality assurance of the Scalable Brain Atlas

Electronic supplementary material The online version of this article (doi:10.1007/s12021-014-9258-x) contains supplementary material, which is available to authorized users.

\section{R. Bakker $(\bowtie) \cdot$ P. Tiesinga}

Department of Neuroinformatics, Donders Institute for Brain,

Cognition and Behavior, Radboud University Nijmegen,

Nijmegen, Netherlands

e-mail: r.bakker@donders.ru.nl

\section{R. Bakker}

Institute of Neuroscience and Medicine (INM-6) and Institute for Advanced Simulation (IAS-6), Jülich Research Centre and JARA, Jülich, Germany

\section{R. Kötter}

Centre for Neuroscience, Donders Institute for Brain, Cognition and Behavior, Radboud University Nijmegen Medical Centre, Nijmegen, The Netherlands specializes in atlas comparisons, searches, coordinate transformations and interactive displays.

Keywords Online Brain Atlas · Comparative anatomy · Macaque $\cdot$ Mouse $\cdot$ Rat $\cdot$ Human $\cdot$ Marmoset $\cdot$ Scalable vector graphics $\cdot$ Structural connectivity $\cdot$ Fiducial points

\section{Introduction}

Brain atlases are used in all areas of neuroscience, and an enormous amount of research data that is tied to coordinates in the brain is produced every day in laboratories worldwide. Many initiatives exist to make these data available through public databases. Federated access to these resources is provided by the Neuroscience Information Framework (NIF, Gardner et al. 2008). The NIF provides services for structured, ontology-based queries, but these are impractical for accessing spatially registered content. For such data, a brain atlasing framework is needed that allows 1) spatial navigation through the brain to select a region or coordinate to initiate a database query, and 2) display of returned results in stereotactic space. Prime examples of existing solutions for these tasks are the Brain Explorer software from the Allen Institute (Sunkin et al. 2013), the (discontinued) Brain Navigator product of Elsevier Inc. (http://brainnav.com), the Java-based Whole Brain Catalog (http://wholebraincatalog.org), the surface-based analysis package Caret (Van Essen 2011), the JuBrain Cytoarchitectonic Atlas Viewer (Mohlberg et al. 2012), the McGill BrainBrowser (https://brainbrowser. cbrain.mcgill.ca/), the NeuroMaps atlas viewer and registration tool (Dubach and Bowden 2009), the Mouse BIRN Atlasing toolkit (Lee et al. 2010), the ThreeDimensional Rodent Atlas System (Hjornevik et al. 2007), 
and the neuroVIISAS integration and simulation platform (Schmitt and Eipert 2012). In principle, each of these products can retrieve and display spatial brain data. What is lacking however is a platform that is 1) not tied to a particular atlas, vendor, database or species, 2) runs in the web browser without having to install software, and 3) allows bilateral interaction with online data resources. The Scalable Brain Atlas (SBA) addresses these issues by using open web standards and having the ambition to contain all publicly available brain atlases that are of sufficient interest to the community.

\section{Web-Based Interactive Brain Atlas}

The SBA has evolved as the successor of the CoCoMacPaxinos-3d tool (CP3D, Bezgin et al. 2009), which is a Java-based platform that volume-renders brain regions taken from the Paxinos rhesus monkey atlas (Paxinos et al. 2000) and displays structural connectivity data from the CoCoMac database (Stephan et al. 2001; Kötter 2004) as directed arrows. While converting CP3D to a fully web-based service, we decided to simplify its $3 \mathrm{~d}$ requirements because support for $3 \mathrm{~d}$ rendering in web browsers is still in its infancy and the required bandwidth restricts its applicability. We instead use a quasi $3 \mathrm{~d}$ approach, whereby sets of $2 \mathrm{~d}$ drawings are stacked together to create a $3 \mathrm{~d}$ experience. Several technologies exist to interactively render such complex drawings inside a web browser, such as Adobe Flash (Adobe Systems Inc.), Microsoft Silverlight (Microsoft Corporation), and Scalable Vector Graphics (SVG, Dahlström et al. 2011). We selected SVG for the SBA because it is an open standard and has broad cross-browser support.

The CP3D tool was tied to a particular species (Macaque) and application (CoCoMac). With the creation of its SVGbased counterpart, we generalized the tool and renamed it to Scalable Brain Atlas. It is scalable because (1) it supports multiple species and multiple brain atlases per species; (2) it has a plugin architecture that allows bidirectional interaction with web-based resources; (3) it is based on SVG. At present, twelve plugins are operational and twenty different brain atlases have been imported. Atlas providers are encouraged to submit data for inclusion. The SBA is hosted at http:// scalablebrainatlas.incf.org.

\section{Core Features of the SBA}

At its core, the SBA has all the features of a typical paper version of a brain atlas, with a $2 \mathrm{~d}$ view of a selected slice and its delineated brain structures (Fig. 1a). It also has a $3 \mathrm{~d}$ panel which contains a stack of all slices and shows the full extent of a selected region (Fig. 1b). A wide variety of mouse actions and keyboard controls are available to navigate, search and display brain regions. The SBA shows the stereotaxic coordinates of the mouse pointer, and markers can be attached to selected locations.

Clicking on a slice in the $3 \mathrm{~d}$ panel opens it in the $2 \mathrm{~d}$ panel. The $2 \mathrm{~d}$ panel shows the brain region delineations in a single slice, which can be underlaid with one of the available imaging modalities. Clicking anywhere in the $2 \mathrm{~d}$ panel triggers a range of actions: (1) The region and all its subparts get highlighted in the $2 \mathrm{~d}$ panel; (2) The region and all its subparts in all slices where the region appears get highlighted in the $3 \mathrm{~d}$ panel. (3) The region gets highlighted in the (hierarchical) list of regions for the given atlas. (4) The active plugin receives a trigger, and can use either the newly selected region or the stereotactic coordinate of the mouse click to update its contents. The $3 \mathrm{~d}$ panel has controls to rotate the view, stretch it in the slice dimension, and to overlay the slice stack with a prerendered $3 \mathrm{~d}$ surface representation of the brain (see $3 \mathrm{dBAR}$ plugin). In addition to this, a framework for displaying stereotaxic markers in the $3 \mathrm{~d}$ panel is available for use by the various plugins.

Twelve plugins are currently included in the SBA core. They are arranged as tiles next to the slice panel (Fig. 1c), and provide functionality such as displaying connectivity derived from the CoCoMac database, getting $3 \mathrm{~d}$ surface renderings of selected brain regions, and showing locations of anatomical landmarks. New plugins can be developed and tested after one-time registration at the SBA server.

In addition to the plugins that interact directly with the graphical interface, the SBA provides web services that allow other websites to retrieve atlas-derived data and images. The most important services are (1) conversion of SVG-based renderings to bitmap images; (2) export of atlas delineations to label volumes that can be analyzed with Matlab; (3) generating a hierarchical list of regions for each imported atlas; (4) computation of several metrics such as region centers and distance matrix. At the most basic level, all available atlas templates are accessible as downloadable files, whose structure is outlined in Appendix 1.

The back end components of the SBA are invisible to the user and include routines to import new sets of atlas data into the system. The SBA works with coronal slices and for each slice the outline of each delineated region needs to be provided as a closed curve. Many atlas sources come in the form of labelled volumes (where the voxel color represents the region name), from which the curves need to be traced.

In the following section we discuss the methods used to create atlas templates, interactive web pages, and plugins. We then present an overview of the plugins and services that are currently available at http://scalablebrainatlas.incf.com. We thereby emphasize how each of them contributes to the goal of the SBA to query online resources that contain brain-region or brain-coordinate related content. In the discussion we highlight the strength of the SBA as a web-based data display engine, and outline further work that would facilitate the data 
integration across atlas templates, across databases, and across species.

\section{Methods}

Importing Atlases into the SBA

The SBA currently contains twenty atlases, listed in Table 1. One of these (PHT00) was available in SVG format from the CP3D project, wherein the SVG polygons were created by manual tracing of scanned atlas pages. For paper atlases that have their document source available, the polygons can be derived automatically if the source is vector-based. Two issues emerge when parsing the file automatically: (1) lines in vector drawings often do not form closed regions; and (2) labels are not always placed inside regions due to space constraints. These issues were largely addressed by the smart parsing methods of Majka et al. (2012), who also proposed a
Common Atlas Format (CAF) to store the processing results and related metadata. We created a filter to import CAF files into the SBA, and used this to import the Marmoset atlas (PWPRT12). Many other high quality print atlases could potentially be imported in this way, but in most cases the copyright has been transferred to the publisher, blocking a public release.

Most of the imported atlases are obtained from label volumes, wherein the color index of each voxel represents the region that it belongs to. Such volumes are typically stored in the NIfTI format (Cox et al. 2004), which has the benefit that the scale and origin of the brain space are included. NIfTI volumes were converted to stacks of coronal bitmap images using the Matlab NIfTI toolbox (http://research.baycrest.org/ $\sim$ jimmy/NIfTI/). We tested several off-the-shelve tools to trace the contours of color coded regions. The open source software potrace (http://potrace.sourceforge.net) is easily integrated into processing pipelines, but the borders of adjacent regions are individually parameterized, which causes small gaps or overlap. These issues are solved by using the 'PowerTrace'

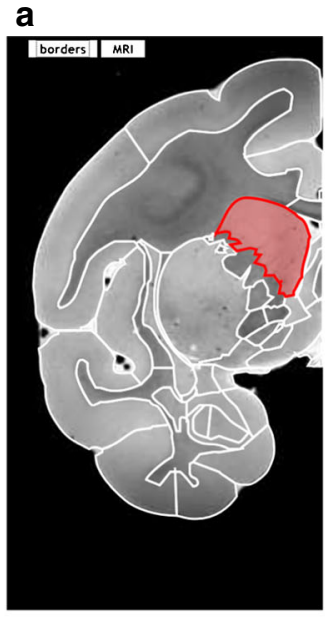

Search: Cd: caudate nucleus

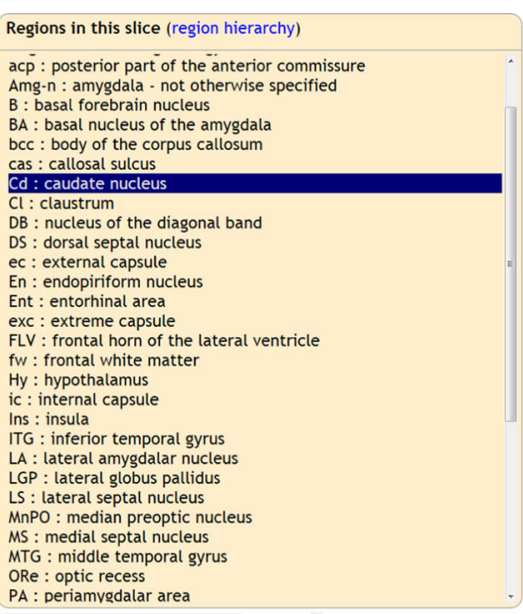

PA : periamygdalar area

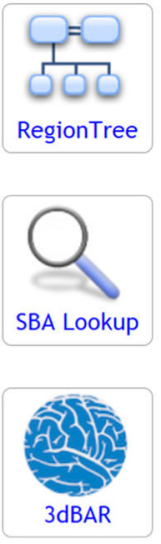
Slice: < $62(308)>1.35 \mathrm{~mm}$ ant. ac

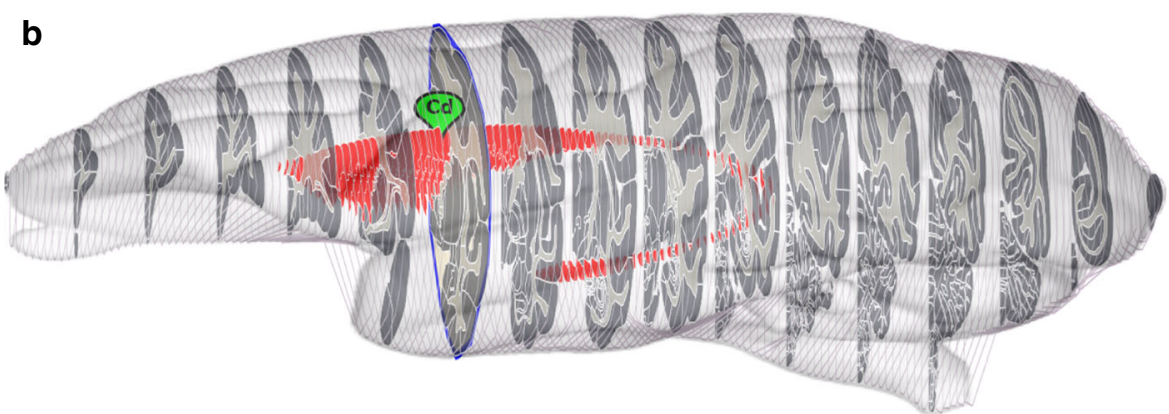

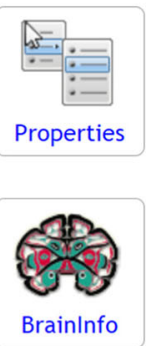
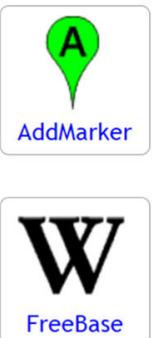

C

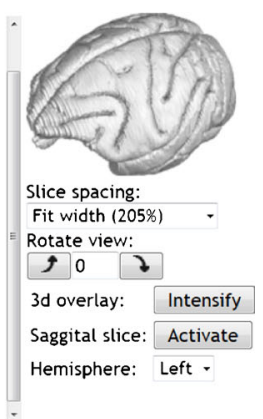

Fig. 1 Components of the atlas viewer: a $2 \mathrm{~d}$ panel displaying a single slice with the parcellation overlaid on the selected imaging modalities, along with a list of structures in the current view or the full region hierarchy (as in Fig. 3); b 3d panel with convex hulls for each slice, and the detailed parcellation for every $10^{\text {th }}$ slice. The current slice is highlighted as a blue contour. By default, the brain is elongated to better show the inside. The $3 \mathrm{~d}$ surface rendering overlay is a static image, generated by the $3 \mathrm{dBAR}$ service (Sec. 5.1), with adjustable transparency; The marker named ' $\mathrm{Cd}$ ' is created by the AddMarker plugin; c Plugin panel. When a plugin gets activated, it responds to changes in selected region, slice and coordinate 
Table 1 Available atlas templates

\begin{tabular}{|c|c|c|c|c|}
\hline Template & Title & Primary publication/site & Parcellation & Imaging modalities \\
\hline \multicolumn{5}{|l|}{ Mouse } \\
\hline ABA2012 & Allen Mouse Brain 2012 & Dong (2008) & 667 areas incl. layer subdivision & Nissl \\
\hline WHS12 & Waxholm Space atlas 2012 & Johnson et al. (2010) & 39 areas & $\begin{array}{l}\text { Nissl and } 21.5 \mu \mathrm{m} \\
\text { resolution } \mathrm{MR} \\
(\mathrm{T} 1, \mathrm{~T} 2 \mathrm{w}, \mathrm{T} 2 *)\end{array}$ \\
\hline \multicolumn{5}{|l|}{ Rat } \\
\hline PLCJB14 & $\begin{array}{l}\text { Waxholm space Sprague } \\
\text { Dawley reference atlas }\end{array}$ & Papp et al. (2014) & $\begin{array}{l}97 \text { areas } \\
\quad(\text { neocortex }=1 \text { area })\end{array}$ & $\begin{array}{l}39 \mu \mathrm{m} \mathrm{T} 2 * \text {, DTI, DWI, } \\
\text { fractional anisotropy }\end{array}$ \\
\hline $\begin{array}{l}\text { CBWJ13 } \\
\text { age_P80 }\end{array}$ & $\begin{array}{l}\text { MR-Histology atlas at } \\
\text { postnatal day } 80\end{array}$ & Calabrese et al. (2013) & 27 areas & $\begin{array}{l}25 \mu \mathrm{m} \text { resolution MR } \\
(\mathrm{T} 2 * / \mathrm{GRE})\end{array}$ \\
\hline VSNetal11 & $\begin{array}{l}\text { Wistar rat in vivo MRI } \\
\text { template }\end{array}$ & Valdés-Hernández et al. (2011) & 129 cortical areas & $\begin{array}{l}\text { T2w, white/gray matter, } \\
\text { csf }\end{array}$ \\
\hline $\begin{array}{l}\text { RMJetal13 } \\
\text { age_P72 }\end{array}$ & $\begin{array}{l}\text { DTI Atlas of the Rat Brain } \\
\text { (age P72) }\end{array}$ & Rumple et al. (2013) & 29 areas & $160 \mu \mathrm{m}$ DTI \\
\hline VLAetal11 & Population-averaged DTI atlas & Veraart et al. (2011) & 14 areas & T1w, DWI, FA \\
\hline \multicolumn{5}{|l|}{ Marmoset } \\
\hline PWPRT12 & $\begin{array}{l}\text { Marmoset Cortical structures } \\
\text { provided by M. Rosa }\end{array}$ & Paxinos et al. (2012) & 116 cortical areas & $\begin{array}{l}\text { Nissl, plus seven other } \\
\text { stains via marmoset- } \\
\text { brain.org }\end{array}$ \\
\hline \multicolumn{5}{|l|}{ Macaque } \\
\hline РHT00 & $\begin{array}{l}\text { Rhesis monkey in stereotaxic } \\
\text { coordinates }\end{array}$ & Paxinos, Huang, Toga (2000) & $\begin{array}{l}283 \text { areas }^{\mathrm{a}}: \text { cortex, amygdala, } \\
\text { thalamus, striatum }\end{array}$ & - \\
\hline DB08 & NeuroMaps Macaque atlas & Dubach, Bowden (2009) & 384 anatomically defined areas & $\mathrm{T} 1$ \\
\hline $\begin{array}{l}\text { FVE91_on } \\
\text { F99 }\end{array}$ & $\begin{array}{l}\text { Felleman and Van Essen } \\
1991 \text { in F99 space }\end{array}$ & $\begin{array}{l}\text { Felleman and Van Essen } \\
\text { (1991) }\end{array}$ & 73 cortical areas & $\mathrm{T} 1$ \\
\hline $\begin{array}{l}\text { LVE00_on_ } \\
\text { F99 }^{\mathrm{b}}\end{array}$ & $\begin{array}{l}\text { Lewis and Van Essen } 2000 \\
\text { in F99 space }\end{array}$ & Lewis, Van Essen (2000) & 87 cortical areas & $\mathrm{T} 1$ \\
\hline $\begin{array}{c}\text { MMFetal11 } \\
\text { on_F99 }^{\mathrm{b}}\end{array}$ & $\begin{array}{l}\text { Markov et al. } 2011 \text { in F99 } \\
\text { space }\end{array}$ & Markov et al. (2011) & 81 cortical areas & $\mathrm{T} 1$ \\
\hline $\begin{array}{l}\text { MERetal12 } \\
\text { on_F99 }^{\mathrm{b}}-\end{array}$ & $\begin{array}{l}\text { Markov et al. } 2012 \text { in F99 } \\
\text { space }\end{array}$ & Markov et al. (2012) & 93 cortical areas & $\mathrm{T} 1$ \\
\hline $\begin{array}{l}\mathrm{RM}_{\mathrm{F}} 9^{\mathrm{b}} \\
\mathrm{b}\end{array}$ & $\begin{array}{l}\text { Regional Map in F99 } \\
\text { space }\end{array}$ & Kötter and Wanke (2005) & 41 anatomical areas & $\mathrm{T} 1$ \\
\hline \multicolumn{5}{|l|}{ Opossum } \\
\hline OPSM14 & $\begin{array}{l}\text { Multimodal atlas of gray short- } \\
\text { tailed opossum brain }\end{array}$ & $\begin{array}{l}\text { Majka et al. (2013a); } \\
\text { Chlodzinska et al. (2013) }\end{array}$ & 105 areas (neocortex $=1$ area) & \\
\hline \multicolumn{5}{|l|}{ Human } \\
\hline EAZ05 & $\begin{array}{l}\text { JuBrain cytoarchitectonic } \\
\text { parcellation }\end{array}$ & Eickhoff et al. (2005) & 76 cyto-architectonic areas & averaged MRI template \\
\hline $\begin{array}{l}\text { LPBA40 } \\
\text { on_SRI24 }\end{array}$ & LBPA40 areas in SRI24 space & $\begin{array}{l}\text { SRI24: Rohlfing et al. (2010) } \\
\text { LBPA40: Shattuck et al. (2008) }\end{array}$ & $\begin{array}{l}56 \text { cortical areas incl. } \\
\text { Left/Right division }\end{array}$ & T1w, T2w, rho \\
\hline $\begin{array}{l}\text { B05_on } \\
\text { Conte } 69\end{array}$ & $\begin{array}{l}\text { Brodmann }{ }^{\mathrm{d}} \text { areas in Conte } 69 \\
\text { space }\end{array}$ & Glasser and Van Essen (2011) & 47 Brodmann cortical areas & $\mathrm{T} 1 \mathrm{w}, \mathrm{T} 2 \mathrm{w}, \mathrm{T} 1 \mathrm{w} / \mathrm{T} 2 \mathrm{w}$ \\
\hline BIGB13 & Bigbrain, resampled at $400 \mu \mathrm{m}$ & Amunts et al (2013) & - & Nissl, resampled at $400 \mu \mathrm{m}$ \\
\hline
\end{tabular}

See http://scalablebrainatlas.incf.org/services/listtemplates.php for the most current list, including templates under development

Abbreviations: MRI Magnetic Resonance Imaging; T1, T1w, T2w, T2*, and rho are MRI contrasts that are sensitive to different tissue properties; GRE Gradient Echo sequence; FA Fractional Anisotropy

${ }^{\mathrm{a}}$ This is a subset of the print atlas, which contains many more subcortical structures

${ }^{\mathrm{b}}$ Obtained as a cortical surface from the SumsDB repository (http://sumsdb.wustl.edu), and converted to volumetric data by assuming a constant cortical thickness of $1.8 \mathrm{~mm}$, using Caret software (Van Essen 2012)

${ }^{\mathrm{c}}$ The F99 space is based on a $0.5 \mathrm{~mm}^{2}$ resolution MR scan (Van Essen 2002)

${ }^{\mathrm{d}}$ Brodmann areas refer to the cytoarchitectonic brain parcellation by Brodmann (1909) 
routines of CorelDraw X4 (Corel Corporation). To prevent PowerTrace from merging regions with similar colors, adjacent regions must be assigned highly contrasting colors.

Six imported atlases are derived from cortical surface parcellations downloaded from the SumsDB brain-mapping repository (Van Essen 2002), processed by the software package Caret (Van Essen 2011). The first step is to convert the labelled surfaces to a label volume, by discretizing the space and assuming a constant cortical thickness; we used $1.8 \mathrm{~mm}$ for Macaque data. In the resulting label volumes, the region boundaries appear 'ragged' (Fig. 2a). We smoothed the regions by first decomposing the volume into individual regions, then applying a blurring filter to each of them, and then regenerating the volume by choosing for each voxel the region with the highest intensity. Figure $2 \mathrm{~b}$ illustrates the smoothing effect of this procedure. The smoothed label volumes were further processed as in the previous paragraph.

\section{Creating Interactive SVG-Based Webpages}

Web browsers have a long tradition in displaying structured text documents, formatted according to the Hypertext Markup Language (HTML) specification (Raggett and Le Hors 1999). This specification deals with text, bitmap images, layout, and hyperlinks. XML (Bray et al. 2008) is the generic container format for languages such as HTML, and SVG is an XMLbased specification for vector graphics. It is supported by all major web browsers.

In an interactive web page, content dynamically responds to keyboard and mouse controls. The response can be: (1) fully client-side, and involve only page elements that are already loaded; or (2) a client-server interaction: retrieve new content from a server and display the result in the client. The SBA plugins use client-server interaction, but in the SBA-core all interactivity is client side. This has the advantage that no internet connection is required once the page is loaded; the downside is that the page may take a while to load; the atlas templates in the SBA are 1 to $2 \mathrm{MB}$ in size, most of which is taken up by the polygons that define region shapes. The server contains a caching mechanism that stores gzip-compressed atlas pages to reduce page load time by about two thirds.

JavaScript (ECMA-262 2011) is the dominant technology to drive client side interactivity. When opening an atlas, the complete set of Javascript Object Notation (JSON) files that define the atlas template (Appendix 1) are downloaded at once and stored in JavaScript memory. JavaScript code then generates a mixture of HTML and SVG, and renders the page in the browser. Inside JavaScript, the page is stored as a hierarchical tree known as the Document Object Model (DOM), which covers both the HTML and SVG elements. Following a mouse click or key press, JavaScript code changes relevant parts of the DOM, which is directly reflected on the displayed page.

Client-server interaction happens when the page is first loaded, and whenever a plugin initiates a web query. Pages typically have a URL that contains an address plus a query that encodes a list of parameters. Many different server-side technologies are available to dynamically respond to these parameters (e.g., the selected atlas template). The SBA uses the PHP scripting language (http://php.net), running on an Apache web server (http://httpd.apache.org/). When opening an atlas, PHP code generates a HTML page that contains a mixture of scripts (JavaScript), data for the selected atlas (JSON), markup (HTML) and style.

\section{Creating Plugins}

The SBA has part of its browser window reserved for plugins, which are small applications that are triggered when the user selects a new region, slice, or stereotaxic coordinate. Following the trigger, the plugin can change the content of its own frame, or it can call routines from the SBA viewer library to change the content of the $2 \mathrm{~d}$ and $3 \mathrm{~d}$ panels of the SBA. For example, it can create a marker using 'new sbaMarker_class(...)', and display it at a stereotaxic location or region center in both the $2 \mathrm{~d}$ and $3 \mathrm{~d}$ panel. An example plugin that prints the text
Fig. 2 Effect of the $3 \mathrm{~d}$ smoothing kernel that is applied to volumes obtained after Surface to Volume conversion in Caret (Van Essen 2011). The blurring kernel is applied separately in each dimension with coefficients $[1,3$, $6,3,1]$. It effectively despeckles the $3 \mathrm{~d}$ volume

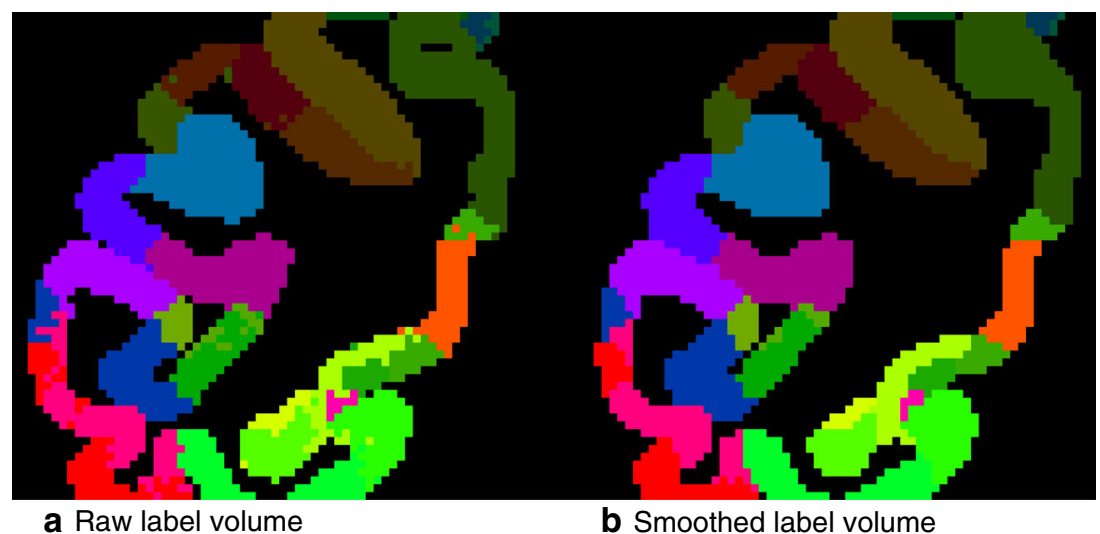


'HELLO WORLD' is listed in Appendix 2, which also provides pointers to the source code of the existing plugins. Writing a plugin does require an understanding of Javascript prototypes.

If no plugin is activated, the plugin window presents a list of all available plugins for the given atlas template. To become part of that list, a plugin has to be approved and hosted at the SBA website.

\section{Bilateral Client-Server Communication}

When a plugin responds to a state change of the atlas viewer, it can use the Javascript httpRequest method to send requests for downloading content. For browser security reasons, Javascript has a same origin policy (SOP), allowing only requests to the server that hosts the website. If a plugin needs to access content from external sites, the solution is to define a PHP script on the SBA server that passes on the request to the external site, and returns the result to the client. This is how it is implemented for the CoCoMac, NeuroLex, Wikipedia and DAI plugins (Sec. 5). Note that the SOP can be bypassed with JSONP (https://en.wikipedia.org/wiki/JSONP), a protocol that disguises data as code, which is exempt from the SOP.

The plugins themselves do not need to be hosted on the SBA server, because the SOP does not apply to Javascript files. Externally hosted plugins can be imported by adding its full URL as a query parameter, for example plugin=http://www.mysite.org/myplugin.js. External plugins must be white-listed on the SBA server to prevent insertion of malicious code.

\section{Creating Services}

Services are scripts designed to serve content to other websites, typically called by a URL and a set of query parameters. The SBA uses a self-documenting service framework: If the service is called with missing parameters, a form is presented with the names and admissible values for these parameters. Each service contains a header section that is used by the sitemap.php service to generate an annotated list of all available services.

\section{Imported Atlas Templates}

An important goal of the SBA is to provide unified access to publicly available atlas templates. To be included, a template must meet four or more of the following criteria:

- $\quad$ is publicly accessible

- is described in a peer-reviewed publication
- contains both a brain parcellation and underlying data modality

- is part of a resource that contains valuable neuroscience data

- is (becoming) a standard reference space

- $\quad$ is available in parseable format (NIfTI-1, CAF)

Table 1 lists the twenty atlas templates that have so far been included, covering six species. The original goal of being a CoCoMac connectivity viewer has caused the Macaque to be overrepresented. Underrepresented are atlases for which copyrights have been transferred to publishers.

\section{Citation Policy}

The SBA processes and integrates atlas templates from many different publicly available sources. If researchers prefer the SBA-processed templates over the original sources, they might be tempted to cite the SBA as the source of an atlas template. To protect the scientific careers of those who created the atlas, the SBA requires its users to always cite the 'defining publications' written by the creators of the template, even when atlases are transformed or combined.

\section{Waxholm Space}

Of special interest are the Waxholm Space templates WHS12 (C57BL/6 mouse) and PLCJB14 (Sprague Dawley rat), promoted by the International Neuroinformatics Coordinating Facility (INCF) as standard reference spaces, and defined by high resolution (21.5 $\mu \mathrm{m}$ isotropic for WHS12) MR imaging data. The purpose of having a standard reference space is to make it easier to transfer data between atlases: if two atlases both have a mapping to the standard space defined, then 1) the mapping between the two atlases is implicitly defined, and data available in each atlases can be displayed together in the standard space. A future role for the SBA can be to recommend standard templates, and to compute optimal (non)linear transforms to and from these standard templates. A preview of this feature is shown in Fig. 3, where the interpeduncular nucleus middle landmark is transformed from the WHS12 to the ABA12 space. The INCF Digital Atlasing Infrastructure (DAI, Hawrylycz et al. 2011) is queried to obtain the transformation between the two templates.

\section{Services}

SBA services are invoked as URL queries, and return formatted content to SBA plugins, clients (end users), or other websites. They typically perform an operation on atlasing data 


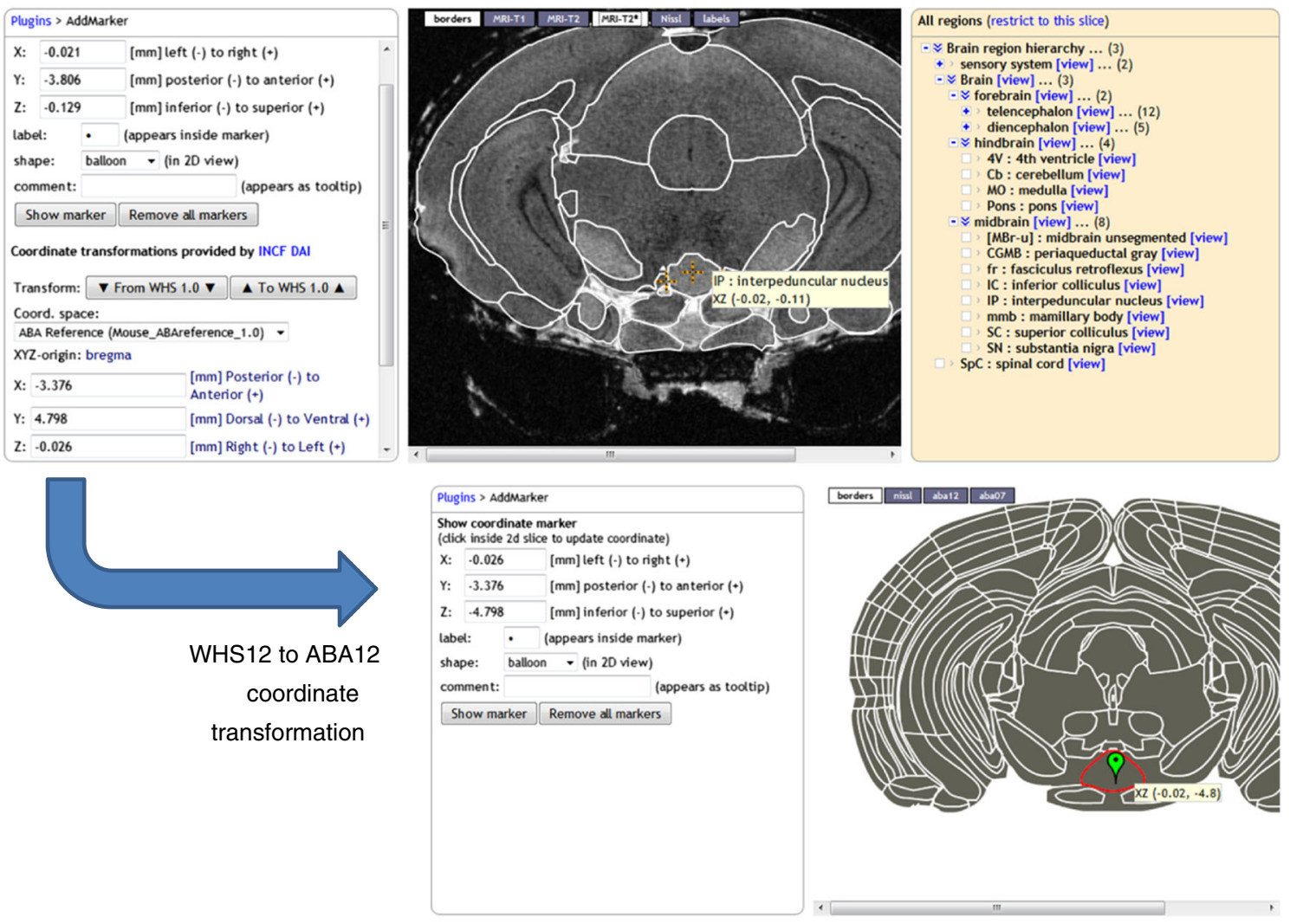

Fig. 3 Coordinate transformation invoked from the AddLandmark plugin, powered by the INCF Digital Atlasing infrastructure. Here, the interpeduncular nucleus landmark is transformed from the WHS12 to the ABA12 template. Table 1 explains template names

within the SBA, and return the result as an image, JSON data, or web page.

The complete list of services is available from the sitemap http://scalablebrainatlas.incf.org/sitemap.php. We here describe six core services, their key parameters and intended use. Services are called as http://scalablebrainatlas.incf.org/ folder/servicename.php?param $1=$ value $1 \&$ param $2=$ value 2 etc. Documentation is displayed when calling a service without parameters.

\section{Atlas Viewer (Main/Coronal3d.php)}

This is the main interactive atlas viewer as described in Sec. 1.2 and displayed in Fig. 1. It has one required parameter template, specifying the atlas template to load. Other useful parameters are:

- region (the brain region to highlight). It is first matched with the list of region abbreviations that comes with the template. If that fails, the alias list is search, then the full names, and finally a case-insensitive match is tried.

- plugin (the plugin to activate). If the plugin starts with http:// or https://, it is assumed that an external plugin is intended. External plugins must be white listed to prevent abuse.
- underlay2d (the image modality to display in the slice panel).

\section{List Atlas Templates (Services/Listtemplates.php)}

Returns an html table with all available atlas templates, the species that they apply to, and the atlas space that the template is registered to. If the atlas space is native, the template defines its own space.

\section{List Atlas Regions (Services/Listregions.php)}

Returns a tab-separated table with all regions defined for the given template. The table includes full names, parent acronyms, and a shape code that specifies whether the region is visible in the atlas viewer.

\section{Coordinate to Region (Services/Coord2region.php)}

Returns the region name that matches the specified stereotactic coordinate, for the given template. The coord parameter specifies a comma separated triplet $\mathrm{x}, \mathrm{y}$ and $\mathrm{z}$. Their origin and direction depends on the template, but we adhere to the NIfTI-1 standard in that $x, y$ and $z$ represent the left/right, 
posterior/anterior and inferior/superior axis, respectively. The service uses ImageMagick (http://www.imagemagick.org) to convert the coronal slice that corresponds to the $y$ parameter to a raster image, looks up the color value of the pixel that corresponds to the $x, z$ location, and finds the corresponding region name in the rgb2acr.json list (see Appendix 1).

\section{Label Volume Service (Services/Rgbslice.php)}

For a given template and slice number, this service generates the SVG representing a coronal slice with color-coded brain regions, and converts it to a raster image if desired. By calling this service for each slice, a web-client can reconstruct the label volume. Matlab (The MathWorks Inc.) scripts are provided to download a complete atlas and visualize individual brain regions, as illustrated in Fig. 4a for template PHT00 and region $\mathrm{V} 1$.

\section{Thumbnail Service}

For a given template and brain region, this service generates thumbnail images that can be used by other websites to illustrate what a brain region looks like and where in the brain it is located. Demand for this service came from the NeuroLex online semantic wiki for neuroscience terms (Larson and Martone 2013). The service provides a choice of thumbnail layouts and image sizes. The output for the combined $2 \mathrm{~d}$ and $3 \mathrm{~d}$ view is illustrated in Fig. $4 \mathrm{~b}$.

\section{Plugins}

This section describes eight general purpose plugins, implemented in Javascript, often with an additional request handler service that resides on the SBA host (see Creating plugins). Some templates have additional plugins available, for example to see original data sets in high resolution.

Three-Dimensional Brain Atlas Reconstructor (3dBAR)

This plugin enables the user to view three-dimensional reconstructions of brain regions from 3dbar.org (Majka et al. 2013b), as illustrated in Fig. 4c for area PHT00-V1. To achieve this, data exchange routines were created to allow the SBA to import atlas templates from the 3dBAR-native CAF format, and $3 \mathrm{dBAR}$ to import templates directly from the SBA. The plugin shows precomputed thumbnails, and links to the $3 \mathrm{dBAR}$ a

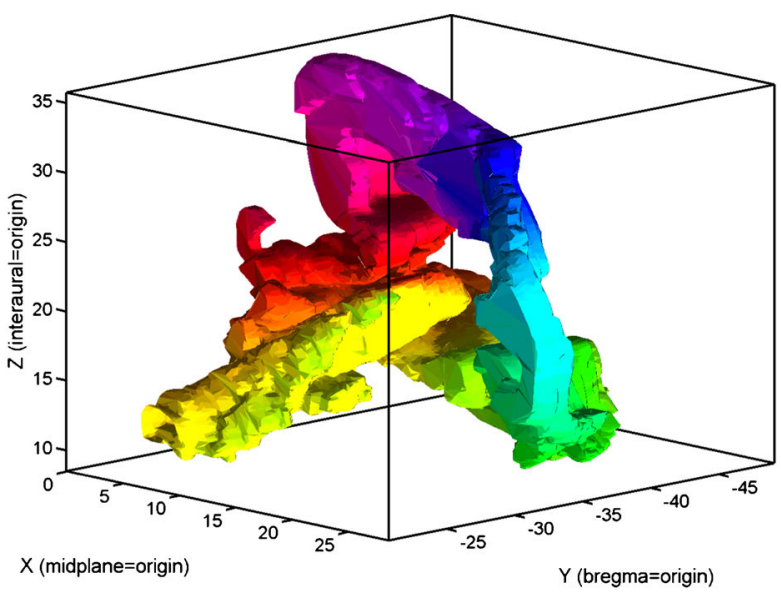

c
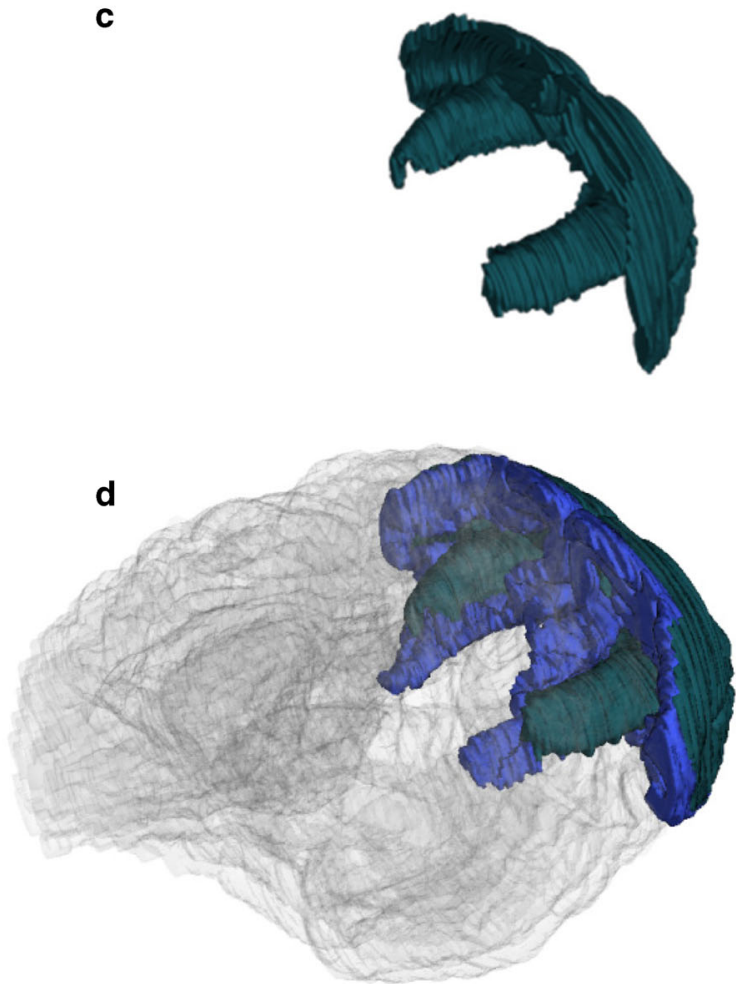

Fig. 4 Various ways to interact with brain region shapes, with PHT00V2 as an example: a Using Matlab functions to download a template, extract a region mask and display it (scripts at http://scalablebrainatlas. incf.org/howto/analyze_templates_in_matlab.php); b Using the SBA thumbnail service; c Using the $3 \mathrm{dBAR}$ plugin from within SBA; d Using the $3 \mathrm{dBAR}$ custom reconstruction service (service.3dbar.org), showing both hemispheres, two areas $(\mathrm{V} 1, \mathrm{~V} 2)$ and a transparent whole brain 
service where the user can construct complex threedimensional scenes, as illustrated in Fig. 4d.

\section{Neuroscience Lexicon (NeuroLex)}

NeuroLex (Larson and Martone 2013) is an online semantic wiki that aims to be the most complete and up to date reference work on neuroscience terms and concepts. It is a component of the NIF. For many brain regions it contains a definition and names of subregions, superregions etc. This plugin actively checks whether the selected brain region in the SBA has a representation in NeuroLex and if so, it downloads and presents the available properties, as illustrated in Fig. 5a for the Thalamus. NeuroLex in turn uses the SBA thumbnail service to graphically display the region.

\section{BrainInfo and NeuroNames}

http://www.braininfo.org is a web portal that contains detailed information on brain sites that are part of the NeuroNames ontology (Bowden et al. 2012). It contains data in the categories: Synonyms, Internal Structure, Cell types, Genes expressed, Locus in brain hierarchy, Connections, and Models. The plugin checks whether BrainInfo has a page about the currently selected brain region. BrainInfo does not currently have a service that returns structured data, and therefore the plugin is limited to displaying links to the corresponding page.

\section{Wikipedia}

Wikipedia (http://en.wikipedia.org) is a collaboratively edited, Internet encyclopedia that contains over 4 million articles in English. The plugin dynamically displays Wikipedia content that matches the full name of the currently selected brain region. Unlike NeuroLex, Wikipedia does not have attributes to limit results to neuroscience terms, and ambiguities with non-neuroscience terms may arise.

\section{Stereotactic Markers and Transformations (AddMarker)}

This plugin enables the placement of visible markers at a given stereotactic location, and displays the location of the last mouse click. For the WHS12 template, the plugin has the additional functionality of transforming coordinates to corresponding locations in other mouse atlas templates. The transformations are provided by the INCF Digital Atlasing Infrastructure (DAI, Hawrylycz et al. 2011). The result is illustrated in Fig. 3 where the center of the Interpeduncular nucleus in the WHS12 template is transformed to the Allen mouse reference atlas (ABA12).

\section{Macaque Connectivity (CoCoMac)}

This plugin demonstrates the SBA at its full potential. It downloads Macaque structural connectivity data from the new CoCoMac database (Bakker et al. 2012) for the selected region, and displays each axonal projection as a marker positioned at the center of the connected region. Figure 6 shows the outgoing projections of region PHT00-25 as a set of markers in the $3 \mathrm{~d}$ panel, as a table in the plugin panel, and in detail on the CoCoMac.g-node.org website, where each projection can be tracked down to the publications in which they were reported.

\section{Brain Region Lookup (SBA Lookup)}

With the growing coverage of species and atlas templates, SBA is becoming a resource of its own. This plugin searches all templates for regions that have the same acronym, full name or alias as the currently selected structure, and provides direct links to the corresponding SBA pages. Figure 5b shows a

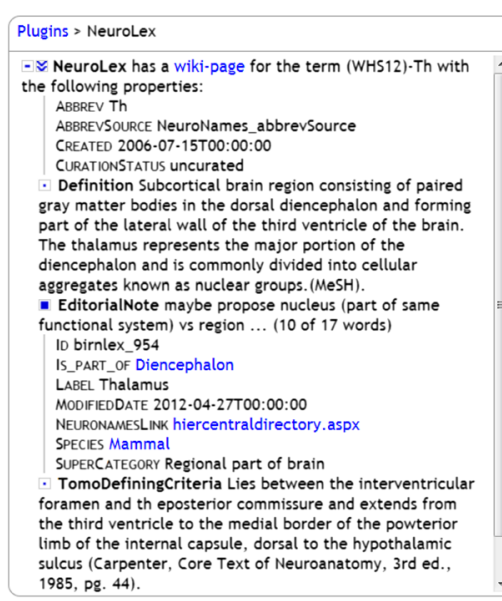

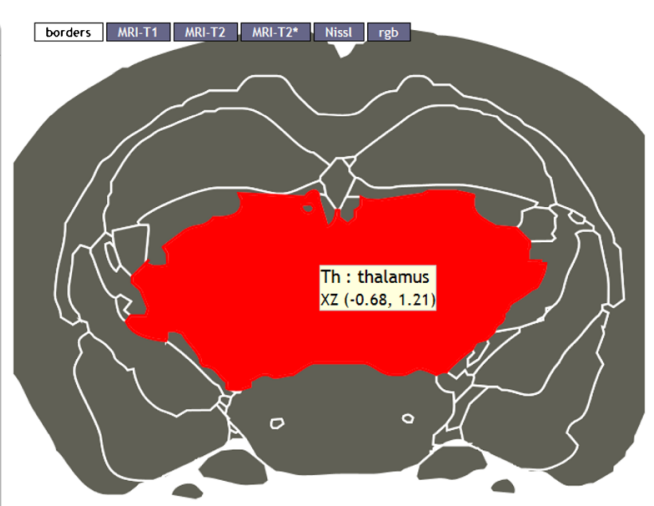

b

\begin{tabular}{|c|c|c|}
\hline \multicolumn{3}{|l|}{ Plugins > SBA Lookup } \\
\hline \multicolumn{3}{|c|}{ The region Th appears in the following templates: } \\
\hline Template & Abbrev. & Full name \\
\hline $\begin{array}{l}\text { WHS09 } \\
\text { (Mouse) }\end{array}$ & Th & Thalamus \\
\hline $\begin{array}{l}\text { PHT00_on_F99 } \\
\text { (Rhesus Monkey) }\end{array}$ & $\mathrm{TH}$ & temporal area $\mathrm{TH}$ \\
\hline $\begin{array}{l}\text { DB08 } \\
\text { (Macaque) }\end{array}$ & Th & thalamus \\
\hline $\begin{array}{l}\text { FVE91_on_F99 } \\
\text { (Macaque) }\end{array}$ & $\mathrm{TH}$ & temporal area $\mathrm{TH}$ \\
\hline $\begin{array}{l}\text { PHT00 } \\
\text { (Macaque) }\end{array}$ & $\mathrm{TH}$ & temporal area $\mathrm{TH}$ \\
\hline $\begin{array}{l}\text { PWPRT12 } \\
\text { (Marmoset) }\end{array}$ & $\mathrm{TH}$ & temporal area $\mathrm{TH}$ \\
\hline \begin{tabular}{|l|} 
ABA12 \\
(Mouse)
\end{tabular} & $\mathrm{TH}$ & Thalamus \\
\hline $\begin{array}{l}\text { ABA07 } \\
\text { (Mouse) }\end{array}$ & $\mathrm{TH}$ & Thalamus \\
\hline $\begin{array}{l}\begin{array}{l}\text { LHAetal07 } \\
\text { (Mouse) }\end{array} \\
\end{array}$ & $\mathrm{TH}$ & Thalamus \\
\hline $\begin{array}{l}\text { WHS12 } \\
\text { (Mouse) }\end{array}$ & Th & thalamus \\
\hline
\end{tabular}

Fig. 5 Output of the NeuroLex (a) and SBA Lookup (b) plugins, both with Th (Thalamus) as the selected region 


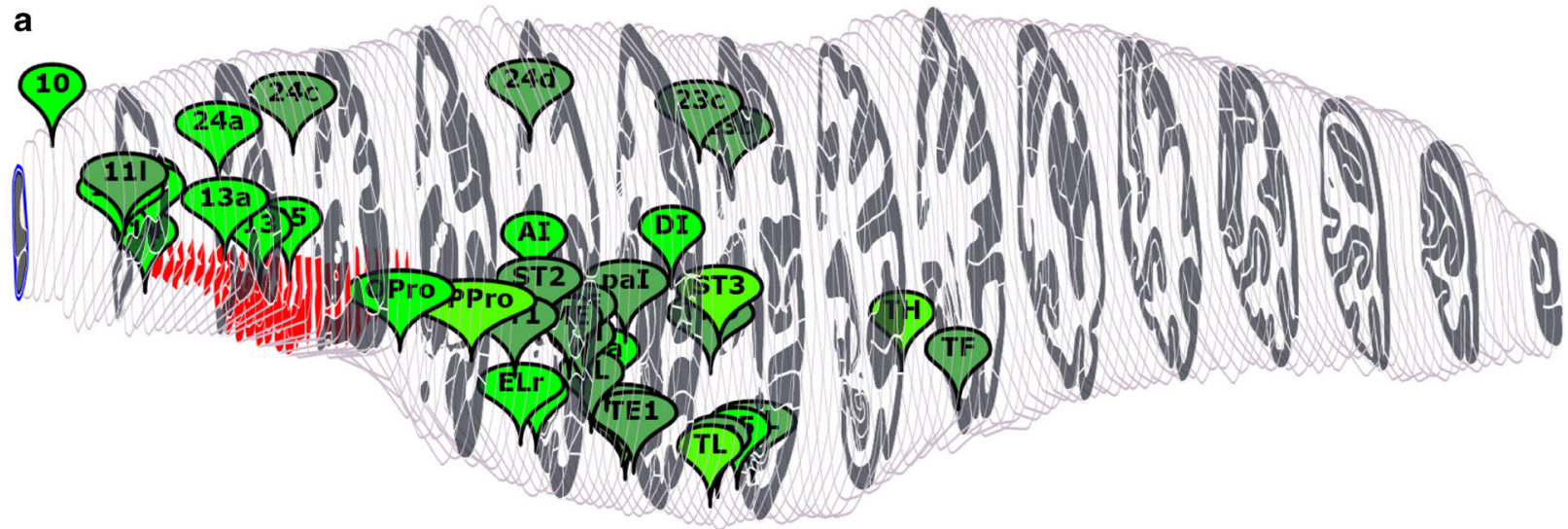

Selected region: 25 : area 25 of cortex

b

\begin{tabular}{|c|c|c|}
\hline \multicolumn{3}{|c|}{ Plugins > CoCoMac } \\
\hline \multicolumn{3}{|c|}{ Axonal projections for region PHTO0-25 } \\
\hline \begin{tabular}{|l|} 
Axon \\
terminal
\end{tabular} & \begin{tabular}{|l|} 
Est. \\
density
\end{tabular} & Density vector \\
\hline $\mathrm{TH}$ & 1.7 & $X X X X X X X X 33 X X X X X X X X X 0 X 0$ \\
\hline ST3 & 1.6 & $x \times X X 3 \times 2 X X 22 X X 3 X$ \\
\hline$T L$ & 1.5 & $X X X X X X X X X X X X 3332312 X 2332211113331122$ \\
\hline 36 & 1.5 & XXXXXXXXXXXX3332312X2332211113331122: \\
\hline TPPro & 1.5 & XXXXXX33212221132211 \\
\hline 25 & 1.4 & 3233333133333333333333333333 XXXXXXXX \\
\hline 13 & 1.4 & 223213333332123113311112222220022113 \\
\hline 14 & 1.4 & $3233133333333333333 X X X X 31122333322133$ \\
\hline 32 & 1.4 & 3313333333 XXXXXXXXXXX333333XX221111 \\
\hline 11 & 1.4 & 1332XXXX22333333111111113311223331333 \\
\hline DI & 1.4 & $2231333333311122 \times X 33333133303311 \times 11$ \\
\hline $11 \mathrm{~m}$ & 1.4 & $3321111332233333333 X X$ \\
\hline $13 a$ & 1.4 & 233222233111333 \\
\hline 10 & 1.3 & 3333333332111100332211 \\
\hline Al & 1.3 & 23333331133333333111 \\
\hline OPro & 1.3 & 122113333 \\
\hline EL & 1.3 & $x \times 3 x$ \\
\hline $24 a$ & 1.2 & 23 \\
\hline
\end{tabular}

Search: 25 : area 25 of cortex

C

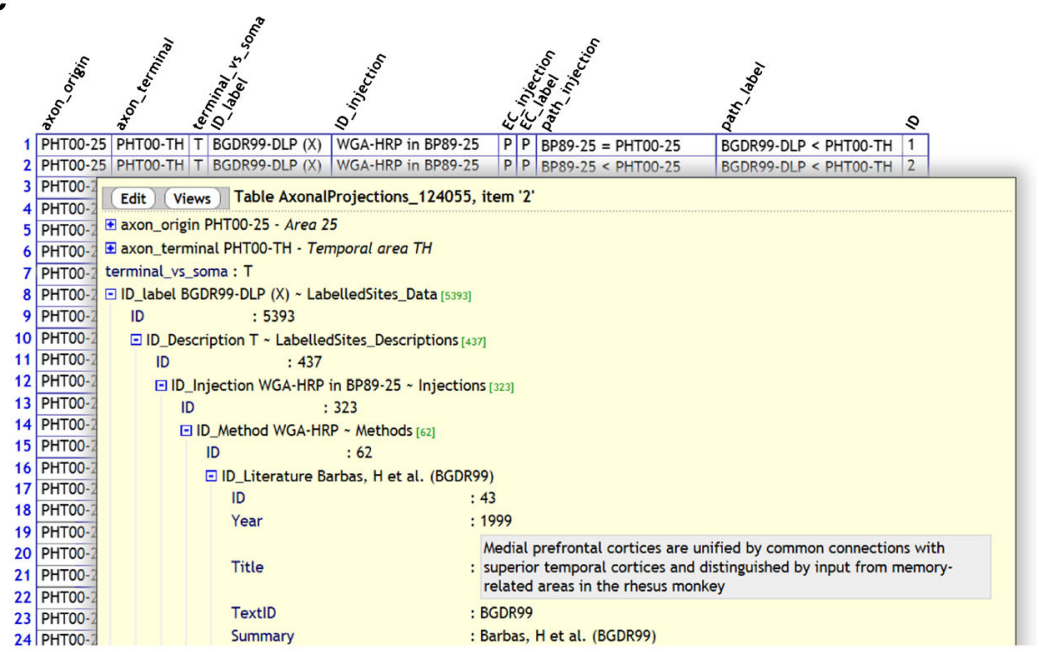

string. Each character is a separate 'piece of evidence', whereby $\mathrm{X}, 0,1,2,3$ mean unknown strength, absent, weak, medium and strong tracer labelling, respectively; c Interactive tabular display at the CoCoMac.gnode.org website allows traceback to the original publication

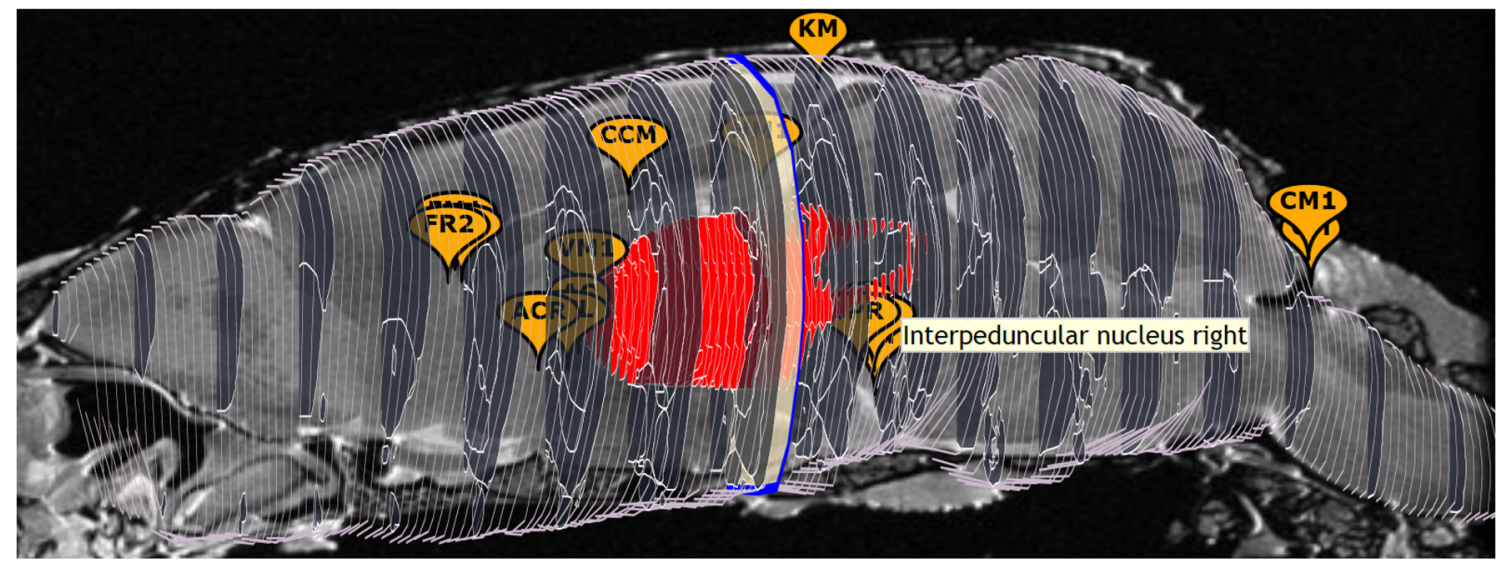

Fig. 7 Sixteen fiducial points, shown by the Landmarks plugin in the $3 \mathrm{~d}$ panel of the WHS12 template, overlaid on the mid-saggital T1 slice. Semi-occluded markers are highlighted on mouse hover. Abbreviations: CM Cerebellum middle, KM Cortex middle, PM Pontine nucleus middle,
HM Hippocampus middle, IP Interpeduncular nucleus middle, IPL/IPR Interpeduncular nucleus left/right, CCM Corpus Callosum middle, VM Ventricle middle, ACL/ACR Anterior Commissure left/right, FM Frontal middle, FL/FR Frontal left/right, AC Anterior Commissure 
the importance of using the full region names to disambiguate the acronym-based results.

\section{Fiducial Points (Landmarks)}

This WHS12-only plugin presents a set of 16 fiducial points that have been validated to be clearly recognizable on the basis of structural MR scans. Figure 7 displays the $3 \mathrm{~d}$ panel with the landmarks. A workflow is under development to register new whole-brain volumes to the WHS12 reference space on the basis of (a subset of) these 16 landmarks (Sergejeva et al. 2015).

\section{Discussion and Conclusion}

What started as a simple CoCoMac visualization application based on manually redrawn region shapes, has grown into a comprehensive web toolkit that supports multiple species, multiple atlases, (third party) plugins, a self-search engine (SBA Lookup) and the ambition to expose all public atlasing resources that are of sufficiently high quality in a public, webbased interface. It attracts about 300 unique visitors per week. The SBA has made a first step towards the integration of data across templates and species with the 'SBA Lookup' plugin. The site is actively maintained, and four new services that will increase interaction and data integration are under way.

The first development is to support the display of saggital and axial slices in the $3 \mathrm{~d}$ panel. A preview of this feature is show in Fig. 7, where the mid-saggital slice is combined with coronal SVG region contours.

The second development is a fully automated pipeline to import new atlas templates. The major hurdle was that the tools to vectorize multi-label images either produce poor quality results or need a manual curation step. This obstacle has recently been cleared with the development of the vectorization tool mindthegap (Kohli et al. 2014).

The third development will combine the automated atlas template pipeline with a nonlinear image registration step. This will superceed the current coordinate transformations as shown in Fig. 3. It will allow users who have volumetric data (MRI volume or Nissl stack) to view their data in conjunction with a (nonlinearly warped) region parcellation from one of the SBA templates. The inverse scenario, whereby a userprovided volume is warped to fit in an existing SBA template, will also be supported. Harder to achieve is the registration of user-proveded single-slice data. A landmark-based workflow (Sec. 5.8) will allow rough positioning of the slice, but more accurate results require an image server that reslices brain volumes at arbitrary angles. While the technology to do so exists (Gustafson et al. 2007) this is beyond the current scope of SBA. For mouse and macaque, we recommend the NeuroMaps Mapper service (http://neuromaps.braininfo.org).

The fourth development is that SBA will be equipped to host atlases at a resolution of up to 2000 pixels in each dimension. At present, SBA does not store such data, but rather displays downsampled images with about $500 \times 500$ pixels in the non-coronal, and 180 pixels in the coronal dimension. High resolution data is only available through plugins that link to external resources. A prototype deep zoom plugin has been developed (http://scalablebrainatlas.incf.org/ABA12?plugin= imaging). It enables responsive display of high resolution content and will make the SBA suitable as a primary host for newly developed atlases.

An obvious omission from the SBA are several popular atlases that have previously appeared in print. There is no technical limitation to import such atlases, but the practice of transferring copyrights to the publishers prevents us from parsing such content. We try to convince copyright owners to become partners in the SBA project.

To conclude, it is our hope that this publication generates new initiatives for plugins, and we look forward to support inclusion of them in SBA. One idea for a community plugin is to have all regions in all supported templates mapped to a common ontology, such as the one developed by Puelles et al. (2013) or NeuroNames (Bowden et al. 2012). We will continue to develop our 'flagship' CoCoMac plugin with new levels of interactivity.

We invite owners of atlasing data to contribute and turn the Scalable Brain Atlas into a community driven resource.

\section{Information Sharing Statement}

All services of the Scalable Brain Atlas (RRID:nlx 98156) are accessible through the url http://scalablebrainatlas.incf.org. The source code for the Scalable Brain Atlas web services is available at https://github.com/INCF/Scalable-Brain-Atlas. The source data for each template can be downloaded as a set of JSON files described in Appendix 1, license restrictions from the respective data owners do apply. Code related to importing new atlas templates is partly based on commercial software and is available on request. An open source release is in preparation, its 'mind-the-gap' vectorization engine is already available at https://github.com/INCF/Vectorization-of-brain-atlases.

Acknowledgments The Scalable Brain Atlas is developed with joint financial support from the International Neuroinformatics Coordinating Facility (INCF) and the Donders Institute for Brain, Cognition and Behaviour of the Radboud University and UMC Nijmegen. The CoCoMac plugin is supported by the German INCF Node (BMBF grant 01GQ0801), Helmholtz Association HASB and portfolio theme SMHB. JUGENE Grant JINB33, and EU Grant 269921 (BrainScaleS). The work was conducted in the context of two INCF Programs: Ontologies of Neural Structures (PONS) and Digital Brain Atlasing (DAI). Inclusion of the Waxholm Space rat template was supported by the European Union Seventh Framework Programme (FP7/2007-2013) under grant agreement 
$\mathrm{n}^{\circ} 604102$ (HBP). Development of image registration services was supported by the Netherlands eSciene Center, grant 027.011.304. The following people contributed to the services, plugins and templates (see Table 1 for abbreviations) that constitute the SBA: Daniel Wojcik and Piotr Majka ( $3 \mathrm{dBAR}$ plugin, whole brain $3 \mathrm{~d}$ renderings, Marmoset template), Andreas Hess and Marina Sergejeva (Landmarks plugin), Hironobu Tokuno, Marcello Rosa and Tristan Chaplin (Marmoset template), Thomas Wachtler, Markus Diesmann (CoCoMac plugin), Jyl Boline, Janis Breeze (INCF taskforce integration), Doug Bowden (DB08 template, NeuroNames expertise), Gleb Bezgin (PHT00 and RM on F99 template and inspiration), Simon Eickhoff (EAZ05 template), Allan Johnson, Seth Ruffins (WHS template), Stephen Larson, Maryann Martone (bidirectional NeuroLex plugin), David van Essen (templates derived from Caret/SumsDB), Jan Sijbers and Jelle Veraart (VLAetal11 template), Henry Kennedy (citation policy).

Conflict of Interest The authors have no conflict of interest

\section{Appendix 2: Example Plugin Code}

The code below shows the code to create a very basic plugin that prints the text "Hello World", and a list of SBA state variables that the plugin can use to initiate queries. This code is available for download from the plugins directory of the SBA: http://scalablebrainatlas.incf.org/plugins/example_ plugin.js.

All other plugins are also in that directory, under the name pluginname plugin.js, where pluginname is the all-lowercase name of the plugin.

$$
\text { /* }
$$

* Plugins are loaded after the global sbaViewer object has been

* initialized, see../js/sba_viewer.js

for available methods of

* the sbaViewer_class

*/

// Plugin constructor.

// Replace example by your own plugin name, in lowercase.

function examplePlugin class (name, sbaViewer) \{

/ / JavaScript's way to call parent constructor

sbaPlugin class.apply (this, [name]) ;

this.niceName = 'myExample' ;

// store the atlas template name (e.g. PHT00) in this.template

this. template = sbaViewer. template ; \}

// JavaScript's way to create a derived class

examplePlugin_class.prototype = new sbaPlugin_class ();

/ / Called when plugin is shown for the first time
examplePlugin class.prototype.activate

= function (sbaviewer, divElem) \{

var sbastate = sbaViewer.getstate () ;

divElem. innerHTML = 'HELLO $\mathrm{WORLD}<\mathrm{p} />^{\prime}+$ json_encode (sbastate);

\}

/*

// Called whenever the state of the SBA has changed

examplePlugin_class.prototype.applystatechange = function (sbaViewer, divElem) \{

// by default, this function calls the activate function,

// you only need to define it when needed for improved efficiency.

\}

* 1

Open Access This article is distributed under the terms of the Creative Commons Attribution License which permits any use, distribution, and reproduction in any medium, provided the original author(s) and the source are credited.

\section{References}

Amunts, K., Lepage, C., Borgeat, L., Mohlberg, H., Dickscheid, T., Rousseau, M. É., Bludau, S., Bazin, P. L., Lewis, L. B., OrosPeusquens, A. M., Shah, N. J., Lippert, T., Zilles, K., \& Evans, A. C. (2013). BigBrain: an ultrahigh-resolution 3D human brain model. Science, 340(6139), 1472-5. doi:10.1126/science.1235381.

Bakker, R., Wachtler, T., \& Diesmann, M. (2012). CoCoMac 2.0 and the future of tract-tracing databases. Frontiers in Neuroinformatics, 6 , 30. doi:10.3389/fninf.2012.00030.

Bezgin, G., Reid, A. T., Schubert, D., \& Kötter, R. (2009). Matching spatial with ontological brain regions using Java tools for visualization, database access, and integrated data analysis. Neuroinformatics, 7, 7-22. doi:10.1007/s12021-008-9039-5.

Bowden, D. M., Song, E., Kosheleva, J., \& Dubach, M. F. (2012). NeuroNames: an ontology for the BrainInfo portal to neuroscience on the web. Neuroinformatics, 10(1), 97-114. doi:10.1007/s12021011-9128-8.

Bray, T., Paoli, J., Sperberg-McQueen, C. M., Maler, E., \& Yergeau, F. (Eds.) (2008) "Extensible Markup Language (XML) 1.0", W3C recommendation http://www.w3.org/TR/2008/REC-xml$20081126 /$.

Brodmann. (1909). "Vergleichende Lokalisationslehre der Grosshirnde" Barth, Leipzig.

Calabrese, E., Badea, A., Watson, C., \& Johnson, G. A. (2013). A quantitative magnetic resonance histology atlas of postnatal rat brain development with regional estimates of growth and variability. NeuroImage, 71, 196-206. doi:10.1016/j.neuroimage.2013.01.017.

Chlodzinska, N., Majka, P., Banasik, T., Djavadian, R. L., Weglarz, W. P., Wójcik, D. K., \& Turlejski, K. (2013). Delineation of brain structures in the Monodelphis domestica opossum brain. 11th International Congress of the Polish Neuroscience Society, Poznań, Poland.

Cox, R. W., Ashburner, J. R. W.,Breman, H., Fissell, F., Haselgrove, C., Holmes, C. J., Lancaster, J. L., Rex, D. E., Smith, S. M., Woodward, 
J. B., \& Strother, S. C. (2004). "A (sort of) new image data format standard: NiFTI-1", poster presented at the OHBM meeting in Budapest, Hungary.

Dahlström, E., Dengler, P., Grasso, A., Lilley, C., McCormack, C., Schepers, D. \& Watt, J. (Eds.) (2011). Scalable Vector Graphics (SVG) 1.1 (Second Edition), http://www.w3.org/TR/2011/RECSVG11-20110816/

Dong, H. W. (2008). "The Allen Reference Atlas: A Digital Color Brain Atlas of the C57BL/6J Male Mouse", Wiley.

Dubach, M. F., \& Bowden, D. M. (2009). "BrainInfo online 3D macaque brain atlas: a database in the shape of a brain," in Society for Neuroscience Annual Meeting, Abstract No. 199.5, Chicago, IL.

ECMA-262 (2011) "ECMAScript Language Specification 5.1 edition", june 2011. http://www.ecma-international.org/publications/files/ ECMA-ST/Ecma-262.pdf.

Eickhoff, S. B., Stephan, K. E., Mohlberg, H., Grefkes, C., Fink, G. R., Amunts, K., \& Zilles, K. (2005). A new SPM toolbox for combining probabilistic cytoarchitectonic maps and functional imaging data. NeuroImage, 25(4), 1325-35. doi:10.1016/j.neuroimage.2004.12. 034.

Felleman, D. J., \& Van Essen, D. C. (1991). Distributed hierarchical processing in the primate cerebral cortex. Cerebral Cortex, 1(1), 1-47. doi:10.1093/cercor/1.1.1.

Gardner, D., Akil, H., Ascoli, G. A., Bowden, D. M., Bug, W., Donohue, D. E., Goldberg, D. H., Grafstein, B., Grethe, J. S., Gupta, A., Halavi, M., Kennedy, D. N., Marenco, L., Martone, M. E., Miller, P. L., Müller, H. M., Robert, A., Shepherd, G. M., Sternberg, P. W., Van Essen, D. C., \& Williams, R. W. (2008). The neuroscience information framework: a data and knowledge environment for neuroscience. Neuroinformatics, 6(3), 149-60. doi:10.1007/s12021008-9024-Z

Glasser, M. F., \& Van Essen, D. C. (2011). Mapping human cortical areas in vivo based on myelin content as revealed by T1-and T2-weighted MRI. Journal of Neuroscience, 31(32), 11597-11616. doi:10.1523/ JNEUROSCI. 2180-11.2011.

Gustafson, C., Bug, W. J., \& Nissanov, J. (2007). NeuroTerrain-a clientserver system for browsing $3 \mathrm{D}$ biomedical image data sets. $B M C$ Bioinformatics, 8, 40. doi:10.1186/1471-2105-8-40.

Hawrylycz, M., Baldock, R. A., Burger, A., Hashikawa, T., Johnson, G. A., Martone, M., Ng, L., Lau, C., Larson, S. D., Nissanov, J., Puelles, L., Ruffins, S., Verbeek, F., Zaslavsky, I., \& Boline, J. (2011). Digital atlasing and standardization in the mouse brain. PLoS Comput Biol 7(2). doi:10.1371/journal.pcbi.1001065.

Hjornevik, T., Leergaard, T. B., Darine, D., Moldestad, O., Dale, A. M., Willoch, F., \& Bjaalie, J. G. (2007) "Three-Dimensional Atlas System for Mouse and Rat Brain Imaging Data". Front Neuroinformatics, 1(4). doi: 10.3389/neuro.11.004.2007.

Johnson, G. A., Badea, A., Brandenburg, J., Cofer, G., Fubara, B., Liu, S., \& Nissanov, J. (2010). Waxholm space: an image-based reference for coordinating mouse brain research. NeuroImage, 53(2), 365-72. doi:10.1016/j.neuroimage.2010.06.067.

Kohli, D., Majka, P., \& Bakker, R. (2014). "Mind the gap - vectorization of brainatlases." https://github.com/INCF/Vectorization-of-brainatlases.

Kötter, R. (2004). Online retrieval, processing, and visualization of primate connectivity data from the CoCoMac database. Neuroinformatics, 2, 127-144. doi:10.1385/NI:2:2:127.

Kötter, R., \& Wanke, E. (2005). Mapping brains without coordinates. Philosophical Transactions of the Royal Society of London. Series B: Biological Sciences, 360(1456), 751-66. doi:10.1098/rstb.2005. 1625.

Larson, S. D., \& Martone, M. E. (2013). NeuroLex.org: an online framework for neuroscience knowledge. Frontiers Neuroinformatics, 7 , 18. doi:10.3389/fninf.2013.00018.

Lee, D., Ruffins, S., Ng, Q., Sane, N., Anderson, S., \& Toga, A. W. (2010). MBAT: a scalable informatics system for unifying digital atlasing workflows. BMC Bioinformatics, 11, 608. doi:10.1186/ 1471-2105-11-608.

Lewis, J.W., Van Essen D.C.(2000). Mapping of architectonic subdivisions in the macaque monkey, with emphasis on parieto-occipital cortex. J Comp Neurol. 4;428(1):79-111. doi:10.1002/10969861(20001204)428:1<79::AID-CNE7>3.0.CO;2-Q

Majka, P., Kublik, E., Furga, G., \& Wójcik, D. K. (2012). Common atlas format and 3D brain atlas reconstructor: infrastructure for constructing 3D brain atlases. Neuroinformatics, 10(2), 181-97. doi:10.1007/ s12021-011-9138-6.

Majka, P., Chlodzinska, N., Banasik, T., Djavadian, R. L., Weglarz, W. P., Turlejski, K., \& Wójcik, D. K. (2013a) "Multimodal stereotactic template of the gray short-tailed opossum's brain" Frontiers conf. abstract Neuroinformatics. doi:10.3389/conf.fninf.2013.09.00021.

Majka, P., Kowalski, J. M., Chlodzinska, N., \& Wójcik, D. K. (2013b). $3 \mathrm{D}$ brain atlas reconstructor service-online repository of threedimensional models of brain structures. Neuroinformatics, 11(4), 507-18. doi:10.1007/s12021-013-9199-9.

Markov, N. T., Misery, P., Falchier, A., Lamy, C., Vezolia, J., Quilodran, R., Giroud, P., Gariel, M. A., Ercsey-Ravasz, M. M., Pilaz, L. J., Huissoud, C., Barone, P., Dehay, C., Toroczkai, Z., Van Essen, D. C., Kennedy, H., \& Knoblauch, K. (2011). Weight consistency specifies regularities of cortical networks. Cerebral Cortex, 21, 1254 1272. doi:10.1093/cercor/bhq201.

Markov, N. T., Ercsey-Ravasz, M. M., Ribeiro Gomes, A. R., Lamy, C., Magrou, L., Vezoli, J., Misery, P., Falchier, A., Quilodran, R., Gariel, M. A., Sallet, J., Gamanut, R., Huissoud, C., Clavagnier, S., Giroud, P., Sappey-Marinier, D., Barone, P., Dehay, C., Toroczkai, Z., Knoblauch, K., Van Essen, D. C., \& Kennedy, H. (2012). A weighted and directed interareal connectivity matrix for macaque cerebral cortex. Cerebral Cortex, 24(1), 17-36. doi:10.1093/cercor/bhs270.

Mohlberg, H., Eickhoff, S. B., Schleicher, A., Zilles, K., \& Amunts, K. (2012). "A new processing pipeline and release of cytoarchitectonic probabilistic maps - JuBrain”, OHBM 2012, Peking, China.

Papp, E. A., Leergaard, T. B., Calabrese, E., Johnson, G. A., \& Bjaalie, J. G. (2014). Waxholm space atlas of the Sprague Dawley rat brain. NeuroImage, 97, 374-386. doi:10.1016/j.neuroimage.2014.04.001.

Paxinos, G., Huang, X.-F., \& Toga, A. W. (2000). "The Rhesus Monkey Brain in Stereotaxic Coordinates" Academic Press.

Paxinos, G., Watson, C., Petrides, M., Rosa, M., \& Tokuno, H. (2012) "The Marmoset Brain in Stereotaxic Coordinates." Academic Press.

Puelles, L., Harrison, M., Paxinos, G., \& Watson, C. (2013). A developmental ontology for the mammalian brain based on the prosomeric model. Trends in Neurosciences, 36(10), 570-8. doi:10.1016/j.tins.2013.06.004.

Raggett, D., Le Hors, A. (Eds.) (1999). "HTML 4.01 Specification", W3C recommendation http://www.w3.org/TR/1999/REChtml401-19991224/.

Rohlfing, T., Zahr, N. M., Sullivan, E. V., \& Pfefferbaum, A. (2010). The SRI24 multichannel atlas of normal adult human brain structure. Human Brain Mapping, 31(5), 798-819. doi:10.1002/hbm.20906.

Rumple, A., McMurray, M., Johns, J., Lauder, J., Makam, P., Radcliffe, M., \& Oguz, I. (2013) "3-dimensional diffusion tensor imaging (DTI) atlas of the rat brain." PLoS One 8(7). doi:10.1371/journal. pone. 0067334

Schmitt, O., \& Eipert, P. (2012). neuroVIISAS: approaching multiscale simulation of the rat connectome. Neuroinformatics, 10(3), 243-67. doi:10.1007/s12021-012-9141-6.

Sergejeva, M., Papp, E. A., Bakker, R., Gaudnek, A., Okamura-Oho, Y., Boline, J., Bjaalie, J. G., \& Hess, A. (2015) “Anatomical landmarks for registration of experimental image data to volumetric rodent brain atlasing templates". J Neurosci Methods, 240, 161-169. doi: 10.1016/j.jneumeth.2014.11.005.

Shattuck, D. W., Mirza, M., Adisetiyo, V., Hojatkashani, C., Salamon, G., Narr, K. L., Poldrack, R. A., Bilder, R. M., \& Toga, A. W. (2008). NeuroImage, 39(3), 1064-1080. doi:10.1016/j.neuroimage. 2007. 09.031 . 
Stephan, K. E., Kamper, L., Bozkurt, A., Burns, G. A. P. C., Young, M. P., \& Kötter, R. (2001). Advanced database methodology for the collation of connectivity data on macaque brain (CoCoMac). Philosophical Transactions of the Royal Society of London. Series B: Biological Sciences, 356, 1159-1186. doi:10.1098/rstb.2001. 0908.

Sunkin, S. M., Ng, L., Lau, C., Dolbeare, T., Gilbert, T. L., Thompson, C. L., Hawrylycz, M., \& Dang, C. (2013). Allen Brain Atlas: an integrated spatio-temporal portal for exploring the central nervous system. Nucleic Acids Research, 41, D996-D1008. doi:10.1093/nar/ gks1042.

Valdés-Hernández, P. A., Sumiyoshi, A., Nonaka, H., Haga, R., AubertVásquez, E., Ogawa, T., Iturria-Medina, Y., Riera, J. J., \& Kawashima, R. (2011). "An in vivo MRI template set for morphometry, tissue segmentation, and fMRI localization in rats" Front Neuroinform, 5. doi:10.3389/fninf.2011.00026

Van Essen, D. C. (2002). Surface-based atlases of cerebellar cortex in the human, macaque, and mouse. Annals of the New York Academy of Sciences, 978, 468-79. doi:10.1111/j.1749-6632.2002.tb07588.x.

Van Essen, D. C. (2011). Cortical cartography and Caret software. NeuroImage, 62(2), 757-64. doi:10.1016/j.neuroimage.2011.10.077.

Van Essen, D.C. (2012) Cortical cartography and Caret software. Neuroimage.15;62(2):757-64 doi:10.1016/j.neuroimage.2011.10.077.

Veraart, J., Leergaard, T. B., Antonsen, B. T., Van Hecke, W., Blockx, I., Jeurissen, B., Jiang, Y., Van der Linden, A., Johnson, G. A., Verhoye, M., \& Sijbers, J. (2011). "Population-averaged diffusion tensor imaging atlas of the Sprague Dawley rat brain." Neuroimage, 58(4). doi:10.1016/j.neuroimage.2011.06.063. 